\title{
PHENIX Studies of the Scaling Properties of Elliptic Flow at RHIC
}

\author{
A. Taranenko for the PHENIX Collaboration $\$$ \\ Department of Chemistry, SUNY, Stony Brook NY 11794 \\ E-mail: arkadij@rcf.rhic.bnl.gov
}

\begin{abstract}
Recent PHENIX elliptic flow $\left(v_{2}\right)$ measurements for identified particles produced in $\mathrm{Au}+\mathrm{Au}$ and $\mathrm{Cu}+\mathrm{Cu}$ collisions at $\sqrt{s_{N N}}=200 \mathrm{GeV}$ are presented and compared to other RHIC measurements. They indicate universal scaling of $v_{2}$ compatible with partonic collectivity leading to the flow of light, strange and heavy quarks with a common expansion velocity field.
\end{abstract}

\section{Introduction}

Universal scaling of elliptic flow $\left(v_{2}\right)$ has been recently observed at RHIC 1, 2, 3. That is, for a broad range of particle species, $v_{2} / n_{q}$ vs. $K E_{T} / n_{q}$ scales to a single function; here, $n_{q}$ and $K E_{T}$ are the number of valence quarks $\left(n_{q}=2,3\right.$ for mesons and baryons respectively) and the transverse kinetic energy of the particle. This observation has been interpreted as evidence that transverse expansion of the matter produced in energetic RHIC collisions, occurs during a phase dominated by partonic collectivity.

In this contribution we further demonstrate; (i) the partonic origin of $v_{2}$ via scaling tests for the $\phi$ meson; (ii) essentially full thermalization for the charm quark via scaling tests for $\mathrm{D}$ meson $v_{2}$; and (iii) validate universal scaling of $v_{2}$ in $\mathrm{Au}+\mathrm{Au}$ and $\mathrm{Cu}+\mathrm{Cu}$ collisions at $\sqrt{s_{N N}}=200 \mathrm{GeV}$.

\section{Further tests for universal scaling of elliptic flow at RHIC}

Differential $v_{2}$ measurements for multi-strange hadrons $(\phi, \Xi, \Omega)$ allow us to address the important question of how the existence of a hadronic phase affects $v_{2}$. These particles are to have a small hadronic cross section for interaction with non-strange hadrons. Consequently, if elliptic flow was established in a phase involving hadrons interacting with their standard hadronic cross sections (post-hadronization), one would expect $v_{2}$ for the multi-strange hadrons to be significantly smaller than that for other hadrons (e.g. $p, \mathrm{~K}, \pi)$. The left panel of Fig. 1 compares PHENIX $v_{2}$ results for the $\phi$ meson, $\pi^{ \pm}, K^{ \pm}$ $\ddagger$ For the full list of PHENIX authors and acknowledgements, see Appendix 'Collaborations' of this volume. 
and $(\bar{p}) p$ from $20-60 \%$ central $\mathrm{Au}+\mathrm{Au}$ collisions; they show that, despite its mass which is comparable to that for the $p$ and $\Lambda$, its $v_{2}\left(K E_{T}\right)$ values are similar to those for the lighter mesons $(\pi$ and $K$ ) whose hadronic re-scattering cross sections are expected to be large. The right panel of Fig. 1 shows the positive results from a validation test for universal scaling of the $\phi$ meson $v_{2}$. The recent high-statistics preliminary STAR results for $\Omega$ and $\Xi$ produced in $\mathrm{Au}+\mathrm{Au}$ collisions at $\sqrt{s_{N N}}=200 \mathrm{GeV}$ [4] show a similar universal scaling [3]. These results suggest that the $v_{2}$ values for hadrons comprised of $\mathrm{u}, \mathrm{d}$ and $\mathrm{s}$ quarks develop in the partonic phase and the existence of a hadronic phase does not lead to their modification.
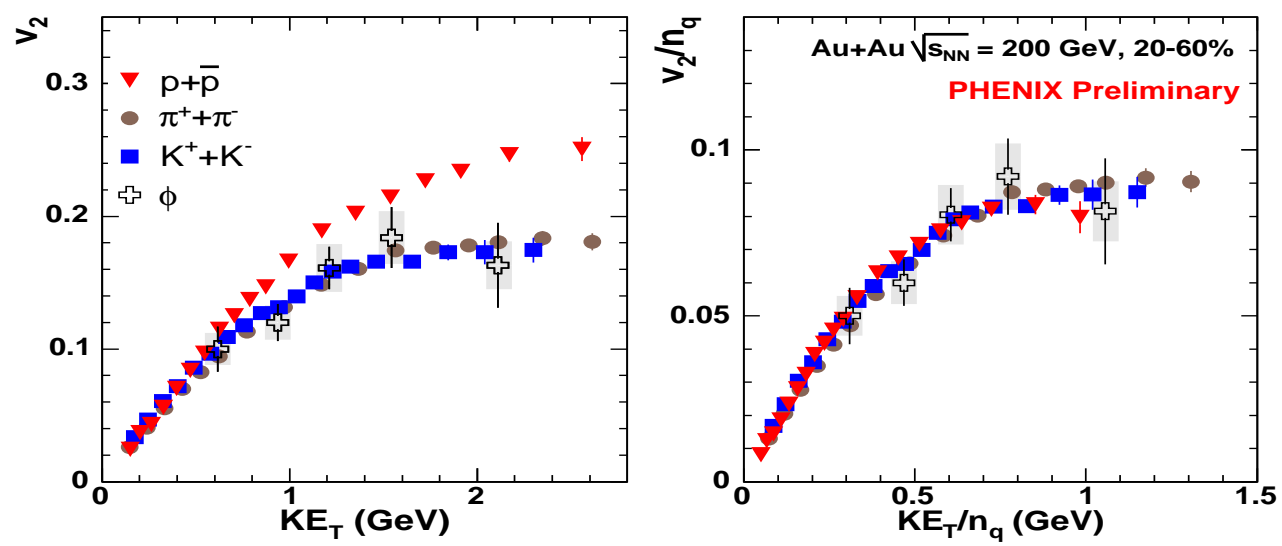

Figure 1: $v_{2}$ vs. $K E_{T}$. and $v_{2} / n_{q}$ vs. $K E_{T} / n_{q}$ for $\pi, K, p$ and $\phi$ mesons detected in semi-central (20-60\%) Au+Au collisions at $\sqrt{s_{N N}}=200 \mathrm{GeV}$.

Further insight into properties of the produced medium at RHIC can be obtained from the study of elliptic flow of particles comprised of heavy quarks (charm or bottom). If charm quarks flow like light quarks, it would indicate an unexpectedly strong interaction of charm with the medium. One method of accessing charm $v_{2}$ is to measure the $v_{2}$ for "non-photonic" electrons [5]. A recent high statistics PHENIX measurements show rather larger elliptic flow for "non-photonic" electrons from $\mathrm{Au}+\mathrm{Au}$ collsions at $\sqrt{s_{N N}}=200 \mathrm{GeV}$ [5]. The "non-photonic" electrons arise mainly from D meson decay for $p_{T}<2 \mathrm{GeV} / \mathrm{c}$. Therefore, the large heavy flavor electron $v_{2}$ indicates that the $\mathrm{D}$ meson and consequently the charm quark, do flow with a large $v_{2}$. This suggests that the in-medium interactions are strong and perhaps frequent enough to thermalize the charm quark. If this is indeed the case, then $v_{2}\left(K E_{T}\right)$ for the $\mathrm{D}$ meson should show the same universal scaling presented earlier for other hadrons.

The $v_{2}$ values for $\mathrm{D}$ mesons can been obtained from the values for non-photonic electrons via detailed simulations, see [5] for details. For these simulations, it is necessary to assume the shape for $v_{2}\left(p_{T}\right)$ for the $\mathrm{D}$ mesons. The left and right panels of Fig. 2 compare the unscaled and scaled results (respectively) for $v_{2}$ vs. $K E_{T}$ for $\pi, K, p$ and $D$ mesons measured in minimum bias $\mathrm{Au}+\mathrm{Au}$ collisions at $\sqrt{s_{N N}}=200 \mathrm{GeV}$. Here, the assumed shape for the $p_{T}$ dependence of the $\mathrm{D}$ meson $v_{2}$ is that for the proton (see 

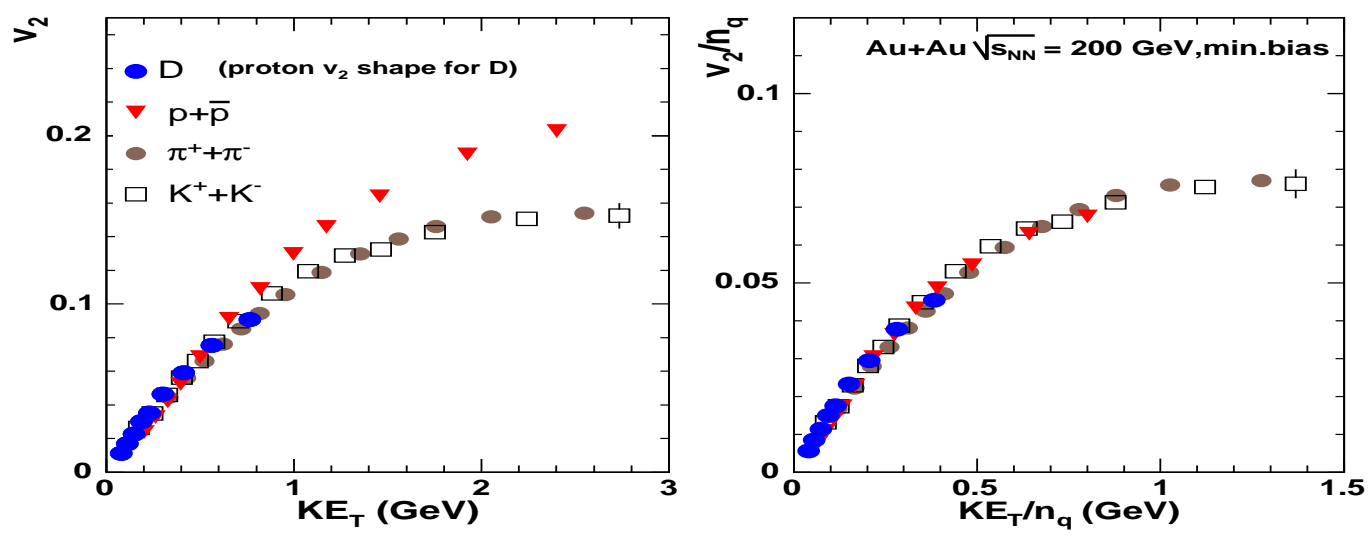

Figure 2: $v_{2}$ vs. $K E_{T}$. and $v_{2} / n_{q}$ vs. $K E_{T} / n_{q}$. for $\pi^{ \pm}, K^{ \pm},(\bar{p}) p$ from minimum bias $\mathrm{Au}+\mathrm{Au}$ collisions at $\sqrt{s_{N N}}=200 \mathrm{GeV}$ [1]. Results for D-meson is shown for the proton-like assumption for the shape of $\mathrm{D} v_{2}\left(p_{T}\right)$ [3, 5]

[3, 5]). This assumption is compatible with a large array of differential $v_{2}$ measurements for different "heavy" particle species [1, 3, 6]. Both panels of Fig. 2 show robust scaling; they confirm that the $\mathrm{D}$ mesons (for $p_{T}<2.0 \mathrm{GeV} / \mathrm{c}$ ) and hence, their associated charm quarks, flow with a fluid velocity field similar to that for the other quarks. This suggests that the medium responds as a thermalized fluid and the transport mean free path is small.

If $v_{2}$ develops in a phase dominated by partonic collectivity and $u, d, s$ and $c$ quarks flow with a common velocity field, one expects universal scaling for all particle species measured at RHIC. Figure 3(a) shows differential flow measurements $v_{2}\left(p_{T}\right)$, for several particle species produced at mid-rapidity in central and semi-central $\mathrm{Au}+\mathrm{Au}$ collisions at $\sqrt{s_{N N}}=200 \mathrm{GeV}$; they span essentially the full range of measurements (several hundred data points) at RHIC. The $v_{2}$ values for pions $\left(\pi^{ \pm}, \pi^{0}\right)$, kaons $K^{ \pm}$, (anti-)protons $(\bar{p}, \mathrm{p})$, (anti-)deuterons $(\bar{d}) \mathrm{d}$ and the $\phi$ meson comprise results from the PHENIX [1, 2, 3, 7]. The values for neutral kaons $\left(K^{0}\right)$, lambdas $(\Lambda+\bar{\Lambda})$, cascades $(\Xi+\bar{\Xi})$ and omegas $(\Omega+\bar{\Omega})$ represent results from the STAR [4, 6]. Fig. 3 (b) shows the scaled results $\left(v_{2} / n_{q} \epsilon\right.$ vs $\left.K E_{T} / n_{q}\right)$ obtained from the same data; here $\epsilon$ is the integral $v_{2}$ of charged hadrons for each of the indicated centrality selections, multiplied by a constant factor $k \sim 3.2$ (i.e. $\epsilon=k \times v_{2}$ ) [1, 2, 3]. Recent measurements [1] indicate that $v_{2}\left(p_{T}\right) / \epsilon$ is independent of centrality and the size of the colliding system as would be expected from a hydrodynamic system. Fig. 3(b) indicates that the relatively complicated dependence of $v_{2}$ on centrality, transverse momentum, particle type, etc., for particles produced at mid-rapidity can be scaled to a single function. Fig. 1(c) demonstrates that the same scaling also holds for $\pi^{ \pm},\left(K^{ \pm}\right)$and $(\bar{p}, \mathrm{p})$ produced at mid-rapidity in $\mathrm{Cu}+\mathrm{Cu}$ collisions at $\sqrt{s_{N N}}=200 \mathrm{GeV}$. 

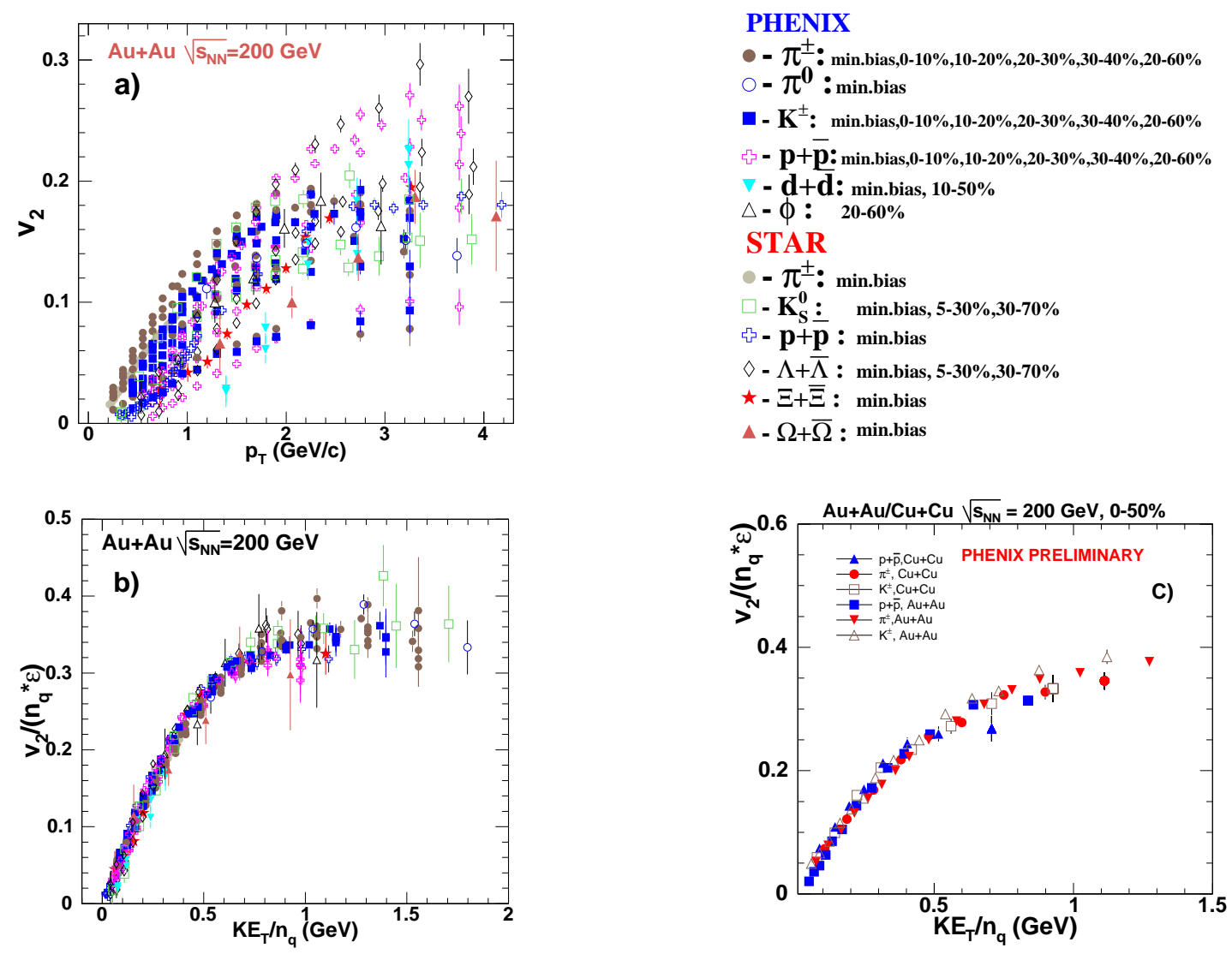

Figure 3: (a) $v_{2}$ vs. $p_{T}$ and (b) $v_{2} /\left(n_{q} \epsilon\right)$ vs. $K E_{T} / n_{q}$ for several centralities and particle species as indicated. (c) $v_{2} /\left(n_{q} \epsilon\right)$ vs. $K E_{T} / n_{q}$ for $\pi^{ \pm}, K^{ \pm}$, and $(\bar{p}, \mathrm{p})$ from $\mathrm{Cu}+\mathrm{Cu}$ and $\mathrm{Au}+\mathrm{Au}$ collisions at $\sqrt{s_{N N}}=200 \mathrm{GeV}$

\section{Summary}

Validation tests for the universal scaling of $v_{2}$ at RHIC, suggest that the transverse expansion dynamics leading to elliptic flow are not controlled by ordinary hadrons interacting with their standard hadronic cross sections. Instead, they suggest a prehardonization state exhibiting partonic collectivity leading to the flow of light, strange and heavy quarks with a common expansion velocity field.

\section{References}

[1] PHENIX Collaboration, A. Adare et.al. nucl-ex/0608033

[2] PHENIX Collaboration, M. Issah et.al. nucl-ex/0604011

[3] R. A. Lacey, A. Taranenko, nucl-ex/0610029

[4] STAR Collaboration, M. Oldenburg et.al., J. Phys. G32, S563 (2006)

[5] PHENIX Collaboration, S. Sakai et.al., J. Phys. G32, S551 (2006); PHENIX Collaboration, A. Adare et.al. nucl-ex/0611018;

[6] STAR Collaboration, J. Adams et.al. Phys. Rev. Lett. 92, 052302 (2004); J. Adams et.al. Phys. Rev. C72, 014904 (2005), J. Adams et.al. Phys. Rev. Lett 95, 122301 (2005)

[7] PHENIX Collaboration, S. S. Adler et.al. Phys. Rev. Lett. 96, 032302 (2006) 